\title{
Hypnotic Drugs: Pharmacological and Therapeutic Issues
}

\author{
Michel Bourin* \\ Neurobiology of anxiety and depression, University of Nantes, 98, rue Joseph Blanchart 44100 Nantes, France
}

Received: December 01, 2017; Accepted: January 18, 2018; Published: January 22, 2018

*Corresponding author: Michel Bourin, Neurobiology of anxiety and depression, University of Nantes, 98, rue Joseph Blanchart 44100 Nantes, France, E-mail: michel.bourin@univ-nantes.fr

\begin{abstract}
The paper proceeds to discuss drugs commonly used in the treatment of sleep disorders, benzodiazepines and newer nonbenzodiazepines compounds. The pharmacokinetic properties of these compounds are briefly described, as are effects of age. Other hypnotics as suvorexant and melatonin are included in this review which concludes with suggestions for the proper identification of patients requiring chemical treatment for sleep disorders and highlights methodologies for choosing the most beneficial pharmacological tools.The following article identifies the appropriate strategies for the prescription of hypnotic drugs.
\end{abstract}

Keys words: Hypnotics; Benzodiazepines; Z Drugs; Suvorexant and Melatonin;

\section{Introduction}

Insomnia is defined as a difficulty falling asleep and / or nocturnal awakening or late night. These sleep disorders are related to various medical or psychological problems. The prevalence of sleep disorders appears to be higher in women than in men. It is also higher in the elderly. Insomnia is usually a symptom rather than an autonomous pathology; it accompanies depressive or anxious syndromes in the majority of cases. The prescription of a hypnotic requires a good knowledge of the circumstances that lead to its use. It is therefore necessary, first of all, to define what is insomnia or more precisely the disorders of sleep. The great problem of the practitioner is therefore to carry out a correct evaluation of the symptoms; this is not very easy but does not dispense with doing it. Most of the time, the prescription of a hypnotic is carried out without this prior procedure thus making it difficult to evaluate [1].

Three groups of drugs used as hypnotics are schematically distinguished

- Allosteric modulators of GABA A receptors (including benzodiazepines)

-Suvorexant which is an antagonist of orexin receptors

- Melatonin which is a natural hormone

\section{Benzodiazepines}

Benzodiazepines have been substituted for barbiturates in the treatment of insomnia due to their lower toxicity. Derivatives marketed ashypnotic's exhibit a relatively rapid resorption rate which allows them to be used as sleep inducers (Table 1).

\begin{tabular}{|c|c|c|}
\hline \multicolumn{2}{|l|}{ Table 1 } & \multicolumn{2}{|l|}{} \\
\hline ICD & Resorption (h) & Half-life (h) \\
\hline Estazolam & $1-1.5$ & $18-24$ \\
\hline Flunitrazepam & $1-1.5$ & $20-30$ \\
\hline Loprazolam & $2-3$ & $8-10$ \\
\hline Nitrazepam & $1-1.5$ & $18-25$ \\
\hline Témazepam & $1.5-2$ & $8-14$ \\
\hline
\end{tabular}

\section{Properties of Benzodiazepines}

Classically, short-acting or long-acting benzodiazepines are separated according to their half-life (mean half-life greater than or less than 10 hours for a hypnotic) [2]. However, this classification has some shortcomings because the elimination half-life is not necessarily the major determinant of the blood concentration as a function of which the hypnotic action ends. Thus, the plasma decay, for a good number of benzodiazepines, is carried out in two phases. The rapid phase is a redistribution of the drug from the perfused tissues, particularly from the brain to the peripheral fats and other weakly perfused tissues (peripheral compartment). The second is a major contributor to decreased plasma concentrations. Products with a half-life of significantly longer than 8 or 10 hours are likely to accumulate and, after several nights of administration, lead to high residual concentrations that may induce diurnal sedation. We must also beware of products whose speed of resorption is not fast, their hypnotic effect is linked to an "overdose", and i.e. the effect is obtained well before the maximum plasma concentration [3].

Whatever the characteristics of benzodiazepine hypnotics, we can note that benzodiazepines with anxiolytic aim can be used in anxiety for a hypnotic purpose by administering a higher dose in the evening, taking into account the rate of resorption often slower [4].

\section{Benzodiazepines have certain advantages [5]:}

- A large margin of safety between therapeutic and toxic 
doses; Thus, in suicide attempts, deaths are exceptional when these derivatives are used alone;

- A paradoxical sleep little affected quantitatively; it seems only to be distributed differently, that is to say, more important at the end of the night;

- Rare drug interactions, but their action appears to be potentiated and / or prolonged with local or general anesthetics, morphine analgesics, antidepressants

\section{There are disadvantages:}

- It is mainly the chronicity of consumption linked to a phenomenon of habituation and the risk of withdrawal incidents during the abrupt cessation of treatment in dependent patients: there are rebounds of insomnia induced by the cessation of treatment, but as well increase of anxiety during the day following the intake.Daily intake should also be avoided, which may also lead to cumulative phenomena leading to sedation in the case of derivatives having a half-life close to 24 hours.

- Memory disorders involving anterograde memory have been described; they occur in all patients as soon as high doses are used, the elderly being particularly sensitive to these effects [6].

- Many benzodiazepines have shown amnesia effects including flunitrazepam and nitrazepam [7]. Since this amnesia can be linked to the plasma peak, it should be made clear to the patient that derivatives with very rapid resorption should only be taken at bedtime. However, amnesia can be observed in the morning on waking or during nocturnal awakenings (aberrant behaviors). Flunitrazepam is also widely used illegally by drug addicts, which has led to the tightening of its prescribing rules [8].

\section{Other hypnotic GABA-A receptor modulators of name the $\mathrm{z}$ drugs}

Close to benzodiazepines, four molecules are now widely used: zolpidem, zopiclone, eszopiclone and zaleplon.

\section{Zolpidem}

It is an imidazopyridine which also binds to the macromolecular site of benzodiazepines. This derivative would have a greater affinity for the benzodiazepine BZ1 receptor subtype than the benzodiazepines themselves, whereas its affinity is lower than the latter for the BZ2 receptor and the BZ3 peripheral. After oral administration, zolpidem has a bioavailability of approximately $70 \%$ with a maximum plasma concentration achieved in 0.5 to 3 hours. At therapeutic doses, its pharmacokinetics is linear. Plasma protein binding is about $92 \%$. The volume of distribution in adults is $0.54 \pm 0.02 \mathrm{l} / \mathrm{kg}$. Zolpidem is eliminated as inactive metabolites (hepatic metabolism), mainly in urine (about $60 \%$ ) and faeces (about 40\%). It has no inducing effect on liver enzymes. The plasma elimination half-life is on average 2.4 hours (0.7 - 3.5 hours) [9].
- In the elderly, decreased hepatic clearance is observed. The concentration at the peak is increased by about $50 \%$ without any significant prolongation of the half-life ( 3 hours on average). The volume of distribution decreases to $0.34 \pm 0.05 \mathrm{l} / \mathrm{kg}$.

- In patients with renal insufficiency, whether dialysed or not, a moderate decrease in clearance is observed. The other kinetic parameters are not modified. Zolpidem is not dialyzable.

- In patients with hepatic impairment, the bioavailability of zolpidem is increased. Its clearance is substantially reduced and the elimination half-life is extended (about)

- Sleep recordings at night showed that zolpidem prolongs stage 2 as well as deep sleep stages 3 and 4.receptors. However, it should be demonstrated that this difference is clinically relevant.

- Adverse effects are related to the individual dose and sensitivity of the patient: dizziness, drowsiness, asthenia, etc. [10]

\section{Zopiclone}

It is a cyclopyrrolone that binds like benzodiazepines to the macromolecular site surrounding the chlorine channel. The absorption of zopiclone is rapid. The maximum concentrations are reached in $1 \mathrm{~h} 30$ to 2 hours and are about 30 and $60 \mathrm{ng} /$ $\mathrm{ml}$ after administration of $3.75 \mathrm{mg}$ and $7.5 \mathrm{mg}$ respectively. The absorption is identical in men and women and is not influenced by the simultaneous ingestion of food or the repetition of doses. The diffusion of zopiclone is very fast from the vascular compartment. Plasma protein binding is low (about 45\%) and is unsaturated. There is a low risk of drug interactions due to protein binding. The volume of distribution is from 91.8 to 104.6 liters. At doses between $3.75 \mathrm{mg}$ and $15 \mathrm{mg}$, plasma clearance is dose independent. The elimination half-life is about 5 hours. After repeated administration, there is no accumulation and inter-individual variations appear to be very low [11].

Zopiclone is mainly metabolized in humans in two major metabolites, zopiclone N-oxide (pharmacologically active in animals) and $\mathrm{N}$-demethyl zopiclone (pharmacologically inactive in animals). An in-vitro study indicates that CYP 3A4 in cytochrome $\mathrm{P} 450$ is the most commonly involved isoenzyme in the metabolism of zopiclone in its metabolites, and that CYP2C8 is also involved in the formation of $\mathrm{N}$-demethyl zopiclone. Their apparent half-lives (evaluated from the urinary data) are approximately $4 \mathrm{~h} 30$ and $1 \mathrm{~h} 30$ respectively. No significant accumulation was observed at repeated doses (15 mg) for 14 days. In animals, no enzymatic induction wasobserved even at high doses. The low renal clearance value of unchangedzopiclone (on average $8.4 \mathrm{ml} / \mathrm{min}$ ) versus plasma clearance $(232 \mathrm{ml}$ / $\mathrm{min}$ ) indicates that the clearance of zopiclone is essentially metabolic. Zopiclone is excreted urine (approximately 80\%) as free metabolites ( $\mathrm{N}$-oxidized and $\mathrm{N}$-demethyl derivatives) and feces (about 16\%).

Sleep laboratory studies show an increase in slow sleep (stage 2) and in deep sleep (stages 3 and 4) [12].

Few side effects have been found. However, some patients 
complain of a taste change with a bitterness of the mouth. Other adverse effects are encountered with other hypnotics: headache, asthenia, and somnolence.

\section{Eszopiclone}

It is the active dextrorotatory stereoisomer of zopiclone. The precise mechanism of action of eszopiclone as a hypnotic is unknown, but its effect isbelieved to result from its interaction with GABA-receptor complexes at binding domains [13].

Eszopiclone is rapidly absorbed after oral administration, with serum levels peaking between 0.45 and 1.3 hours. The elimination half-life of eszopiclone is approximately 6 hours and it is extensively metabolized by oxidation and demethylation. Approximately $52 \%$ to $59 \%$ of a dose is weakly bound to plasma protein. Cytochrome P450 (CYP) isozymes CYP3A4 and CYP2E1 are involved in the biotransformation of eszopiclone; thus, drugs that induce or inhibit these CYP isozymes may affect the metabolism of eszopiclone. Less than $10 \%$ of the orally administered dose is excreted in the urine as racemic zopiclone. In terms of benzodiazepine receptor binding and relevant potency, $3 \mathrm{mg}$ of eszopiclone is equivalent to $10 \mathrm{mg}$ of diazepam [13] Eszopicolone is presenting with the same side effects as zopiclone including bitter taste [15].

\section{Zaleplon}

Zaleplon is a pyrazolopyrimidine which binds to the benzodiazepine binding site on GABA-A receptors containing the alpha 1 receptor sub unit and alters the GABA mediated chloride channel opening. This is responsible for sedative effect of the drug [16]. Zaleplon is rapidly absorbed after oral administration and is distributed in protein bound form $(60 \%)$ It is metabolised in the liver to inactive metabolite and is excreted mainly in urine. The onset of action for Zaleplon is rapid. The half-life of Zaleplon is 1 hour.The side effects of Zaleplonare : amnesia, dizziness , light headedness, somnolence, hallucinations vertigo, palpitation, dry mouth ,nausea , lack of concentrations, paresthesia [17].

For these four molecules, it was shown that, as for benzodiazepines, there were withdrawal syndromes at cessation of treatment and misuse problems as with hypnotic benzodiazepines $[18,19]$.

\section{Suvorexant}

Suvorexant, approved in late 2014 in the United States and Japan for the treatment of insomnia characterized by difficulty achieving and/or maintaining sleep, is a dual orexin receptor antagonist and the first drug in its class to reach the market [20]. Its development followed from the 1998 discovery of orexins (also called hypocretins) [21], excitatory neuropeptides originating from neurons in the hypothalamus involved in regulation of sleep and wake, feeding behavior and energy regulation, motor activity, and reward-seeking behavior. Suvorexant objectively improves sleep, shortening the time to achieve persistent sleep and reducing wake after sleep onset, although at approved doses ( $\leq$ $20 \mathrm{mg}$ ) the benefit was subjectively assessed as modest. Its halflife of 12 hours is relatively long for a modern hypnotic; however, at approved doses ( $\leq 20 \mathrm{mg}$ ) next-day sedation and driving impairment were much less apparent than at higher doses. Suvorexant is metabolized by the hepatic CYP3A system and should be avoided in combination with strong CYP3A inhibitors. Drug levels are higher in women and obese people; hence, dosing should be conservative in obese women. Administration with food delays drug absorption and is not advised. No dose adjustment is needed for advanced age, renal impairment, or mild-to-moderate hepatic impairment [22]. Suvorexant in contraindicated in narcolepsy and has not been studied in children. In alignment with the changes begun in 2013 in the labeling of other hypnotics, the United States Food and Drug Administration advises that the lowest dose effective to treat symptoms be used and that patients be advised of the possibility of next-day impairment in function, including driving. Infrequent but notable side effects included abnormal dreams, sleep paralysis, and suicidal ideation that were dose-related and reported to be mild. Given its mechanism of action, cataplexy and rapid eye movement (REM) sleep behavior disorder could potentially occur in some patients taking this medication [23].

\section{Melatonin}

Melatonin, or 5-methoxy- $\mathrm{N}$-acetyltryptamine, is synthesized and released by the pineal gland and locally in the retina following a circadian rhythm, with low levels during the day and elevated levels at night. Melatonin activates two high-affinity G proteincoupled receptors, termed MT1 and MT2, to exert beneficial actions in sleep and circadian abnormality; it is an endogenous regulator of sleep in man and not stricto sensu a hypnotic [24]. It is marketed in sustained-release form (Circadin ${ }^{\circledR}$, melatonin LP) which is indicated as monotherapy for the short-term treatment of primary insomnia Characterized by poor quality sleep in patients aged 55 years or older [25]. Indeed, in elderly insomniacs, the rate of melatonin is significantly lower than in elderly subjects without sleep problems. Clinical data do not support the assumption that Circadin ${ }^{\circledR}$ provides a clinical benefit in the management of primary insomnia without comorbidity [26] .The recommended dosage is $2 \mathrm{mg}$ once daily, 1 to 2 hours before bedtime and after meals. This dosage may be continued for up to 13 weeks. The safety and efficacy of Circadin in children aged 0 to 18 years has not yet been established.

The effect of renal insufficiency, whatever its degree, on the pharmacokinetics of melatonin has not been studied. Caution should be exercised when administering melatonin to patients with renal insufficiency. No data are currently available on the use of Circadin in patients with hepatic impairment. Published data show that endogenous melatonin levels increase markedly during daytime hours due to reduced clearance in patients with hepatic impairment. Therefore, Circadin is not recommended for patients with hepatic impairment. The tablets should be swallowed whole to preserve their sustained release properties. Do not crush or chew them to make them easier to take.

\section{Conclusion}

The prescription of a hypnotic must not become systematic; this is why the National Institute of Mental Health in the United 
States issued the rules after a consensus conference (Table 2)

The choice of the hypnotic can only be made on the basis of pharmacokinetics, the only criterion of choice that can be exploited at the present time.

In case of prolonged prescription, it is possible to observe a phenomenon of dependence which can lead in the elderly a certain "desocialization".
Continuous treatment should therefore be avoided by prescribing the hypnotic 2 or 3 days a week, on evenings when the subject is most afraid of a bad night. It is necessary, whatever the age of the patient, to determine clearly whether Sleep disorder is not due to an environment or lifestyle that can be altered; Then to specify if it is an insomnia of sleep, nocturnal awakenings, or early morning awakenings. Melatonin appears to be indicated for the re-synchronization of sleep phases.

\section{Table 2: NIH Consensus Conference: Drugs and Insomnia [27]}

1. Transient insomnia (situational stress, jet lag): no treatment orpunctual intake of hypnotics 1 to 3 days

2. Insomnia of recent onset and short duration: hypnotic plus lifestyle and non-medicated therapeutics 3 weeks approx

3. Chronic insomnia (many causes, requires a full medical evaluation a. Initial approach: no drugs and treatment of the underlying physical or psychiatric illness. b. Hypnotic test less than 1 month; if longer, treatment should be intermittent.

\section{References}

1. Matheson E, Hainer BL. Insomnia: Pharmacologic Therapy. Am Fam Physician. 2017;96(1):29-35.

2. Breimer DD, Jochemsen R, von Albert HH. Pharmacokinetics of benzodiazepines. Short-actingversuslong-acting. Arzneimittelforschung. 1980;30(5a):875-881.

3. Greenblatt DJ. Benzodiazepine hypnotics: sorting the pharmacokinetic facts. J Clin Psychiatry. 1991;52 Suppl:4-10.

4. Dailly E, Bourin M. The use of benzodiazepines in the aged patient clinical and pharmacological considerations. Pak J Pharm Sci. 2008;21(2):144-150.

5. Greenblatt DJ. Pharmacology of benzodiazepine hypnotics.J Clin Psychiatry. 1992;53 Suppl:7-13.

6. Bourin M, Briley M. Sedation, an unpleasant, undesirable and potentially dangerous side-effect of many psychotropic drugs. Hum Psychopharmacol. 2004;19(2):135-139.

7. Liebrenz M, Schneider M, Buadze A, Gehring MT, Dube A, Caflisch C. High-Dose Benzodiazepine Users' Perceptions and Experiences of Anterograde Amnesia. J Am Acad Psychiatry Law. 2016;44(3):328-337.

8. Tan KR, Rudolph U, Lüscher C. Hooked on benzodiazepines: GABAA receptor subtypes and addiction. Trends Neurosci. 2011;34(4):188197. doi: 10.1016/j.tins.2011.01.004

9. Monti JM, Spence DW, Buttoo K, Pandi-Perumal SR. Zolpidem's use for insomnia. Asian J Psychiatr. 2017;25:79-90. doi: 10.1016/j. ajp.2016.10.006

10.Ganzoni E, Santoni JP, Chevillard V, Sébille M, Mathy B. Zolpidem in insomnia: a 3-year post-marketing surveillance study in Switzerland. J Int Med Res. 1995;23(1):61-73.

11.Musch B, Maillard F. Zopiclone, the third generation hypnotic: a clinical overview. Int Clin Psychopharmacol. 1990; 5Suppl2:147-158.

12.Noble S, Langtry HD, Lamb HM. Zopiclone. An update of its pharmacology, clinical efficacy and tolerability in the treatment of insomnia. Drugs. 1998;55(2):277-302.

13.Halas CJ. Eszopiclone. Am J Health Syst Pharm. 2006;63(1):41-48.

14.Hair PI, McCormack PL, Curran MP. Eszopiclone: a review of its use in the treatment of insomnia. Drugs. 2008;68(10):1415-1434.
15.Doty RL, Treem J, Tourbier I, Mirza N. A double-blind study of the influences of eszopiclone on dysgeusia and taste function. Pharmacol Biochem Behav. 2009;94(2):312-318. doi: 10.1016/j.pbb.2009.09.011

16.Weitzel KW, Wickman JM, Augustin SG, Strom JG. Zaleplon: a pyrazolopyrimidine sedative-hypnotic agent for the treatment of insomnia. Clin Ther. 2000;22(11):1254-1267.

17.Heydorn WE. Zaleplon - a review of a novel sedative hypnotic used in the treatment of insomnia. Expert Opin Investig Drugs. 2000;9(4):841858.

18.Gunja N. The clinical and forensic toxicology of Z-drugs. J Med Toxicol. 2013;9(2):155-162. doi: 10.1007/s13181-013-0292-0

19.Stranks EK, Crowe SF. The acute cognitive effects of zopiclone, zolpidem, zaleplon, and eszopiclone: a systematic review and meta-analysis. J Clin Exp Neuropsychol. 2014; 36(7):691-700. doi: $10.1080 / 13803395.2014 .928268$

20.0wen RT. Suvorexant: efficacy and safety profile of a dual orexin receptor antagonist in treating insomnia. Drugs Today (Barc) 2016;52(1):29-40. doi: 10.1358/dot.2016.52.1.2439940

21.Jacobson LH, Chen S, Mir S, Hoyer D .Orexin OX2 Receptor Antagonists as Sleep Aids. Curr Top Behav Neurosci. 2017;33:105-136. doi: 10.1007/7854_2016_47

22.Patel KV, Aspesi AV, Evoy KE. Suvorexant: a dual orexin receptor antagonist for the treatment of sleep onset and sleep maintenance insomnia .Ann Pharmacother. 2015;49(4):477-483. doi: $10.1177 / 1060028015570467$

23.Kuriyama A, Tabata H. Suvorexant for the treatment of primary insomnia: A systematic review and meta-analysis. Sleep Med Rev. 2017;35:1-7. doi: 10.1016/j.smrv.2016.09.004

24.Arendt J. Melatonin and human rhythms. Chronobiology Int. 2006;23(1-2):21-37.

25.Wade AG, Ford I, Crawford G, McMahon AD, Nir T, Laudon M, et al. Efficacy of prolonged release melatonin in insomnia patients aged 5580 years: quality of sleep and next-day alertness outcomes. Curr Med Res Opin. 2007;23(10):2597-2605.

26.Lemoine $\mathrm{P}$, Zisapel N. Prolonged-release formulation of melatonin (Circadin) for the treatment of insomnia. Expert Opin Pharmacother. 2012;13(6):895-905. doi: 10.1517/14656566.2012.667076

27.NIH State of the Science conference Statement on Manifestations and Management of Chronic Insomnia in Adults statement 2005. J Clin Sleep Med. 2005;1:412- 423. 